\title{
MINERAL PRODUCTION IN IOWA
}

IN 1908

BY

S. W. BEYER 

MINERAL PRODUCTION IN IOWA FOR 1908*

BY S. W. BEYER.

VALUE OF MINERAL PRODUCTION.

1906

\begin{tabular}{|c|c|}
\hline Coal & $\$ 11,619,455$ \\
\hline 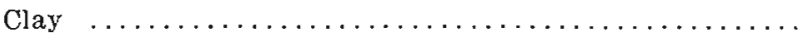 & $: 3,477,237$ \\
\hline 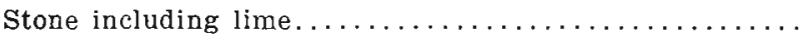 & $5,77,782$ \\
\hline 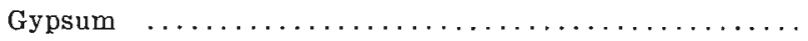 & 573,498 \\
\hline 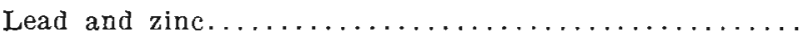 & 26,300 \\
\hline 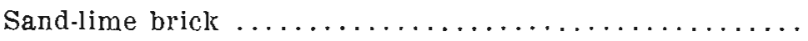 & 38,225 \\
\hline Mineral water & 27,540 \\
\hline 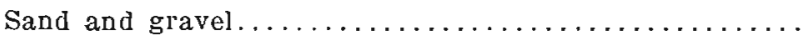 & 74,380 \\
\hline
\end{tabular}

1907

Coal

$\$ 12,258,012$

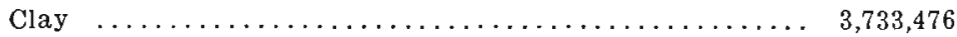

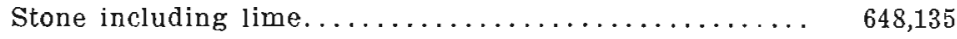

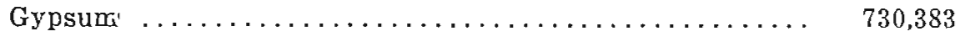

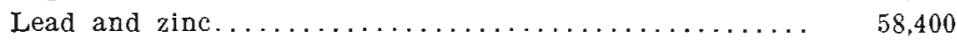

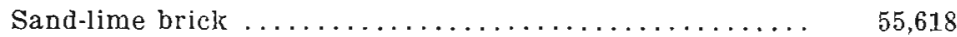

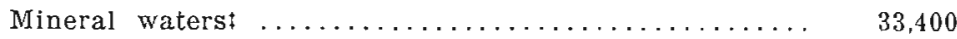

Sand and gravel.......................... 110,501

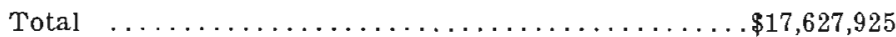

1908

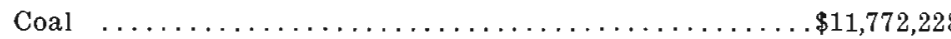

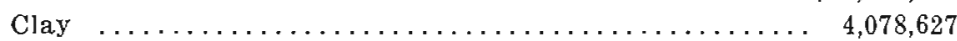

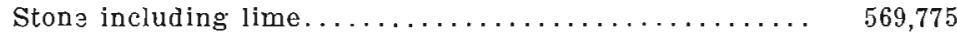

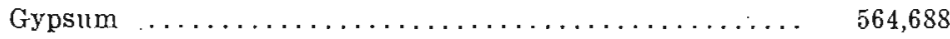

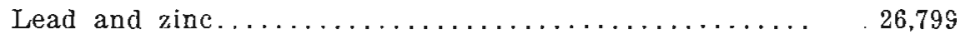

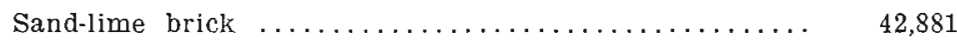

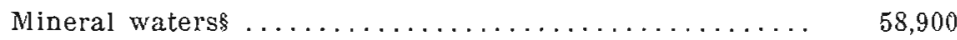

Sand and gravel* $\ldots \ldots \ldots \ldots \ldots \ldots \ldots \ldots \ldots \ldots \ldots \ldots \ldots \ldots \ldots \ldots \ldots, 549$

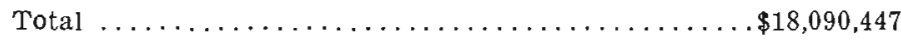

-The mineral statistics for 1908 were collected through co-operation of the Iowa Geological Survey and the United States Geological Survey. The slight differences in results shown below are due to natural variations in interpretation and editing of returns.

Mineral paint is combined with mineral water.

iMineral paints and iron ore are included with mineral waters.

\$Mineral paints included with mineral waters.

**Portland cement included with sand and gravel. 
The mineral production for 1908 shows a gain in the value of clay produots, mineral waters and sand and gravel and a falling off in all of the other mineral products produced in the state. The greatest shrinkage was in the coal production. The total value of mineral products marketed shows a gain due to the opening and operation of the Portland cement plant at Mason City. Prices for all the mineral products of the state, save coal, were lower than for the preceding year. The manifold uses of cement and the greater effort directed toward the betterment of the public highways are responsible for the increase in the production of sand and gravel. The great increase in the manufacture and use of drain tile owing to the continuance of wet years swells the clay output. The outlook for 1909 is favorable for an increased production in all of the more important mineral industries.

Table I gives the total mineral production by counties and follows herewith:

TABLE NO. I.

Value of Total Mineral Production by Counties for 1908.

\begin{tabular}{|c|c|c|c|c|c|c|}
\hline Counties & 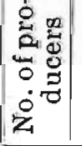 & Coal & Clay & $\begin{array}{l}\text { Stone } \\
\text { and } \\
\text { Lime }\end{array}$ & $\begin{array}{l}\text { Miscel- } \\
\text { laneous }\end{array}$ & $\begin{array}{l}\text { Total } \\
\text { Value }\end{array}$ \\
\hline Adair & 2 & & 7,300 & & & 7,300 \\
\hline Adams & 14 & 42,335 & 25,289 & & $\ldots$ & 67,524 \\
\hline Allamakee & 2 & & & 242 & & 242 \\
\hline Appanoose ....... & 71 & $2,151,905$ & 28,648 & -- & & $2,170,894$ \\
\hline Audubon $\ldots \ldots \ldots$ & 1 & & & & & \\
\hline Benton $\ldots$. & 9 & (n- & 28,674 & 491 & 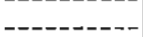 & 29,165 \\
\hline Black Hawlk & 17 & & & 13,405 & $\$ 41,915$ & 69,920 \\
\hline Boone $\ldots . . . \ldots$. & 15 & 461,544 & 53,475 & $-\ldots$ & - & 515,019 \\
\hline Bremer $\ldots \ldots \ldots \ldots$ & & & & & & \\
\hline Buena Vista ... & 4 & --- & 40,000 & -- & ----- & 40,250 \\
\hline Butler ........... & 1 & & & & & \\
\hline Calhoun $\ldots . . . \ldots$ & 3 & - n- & 36,900 & & 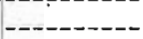 & 36,900 \\
\hline Carroll ........... & 1 & --- & &.--- & -----1 & \\
\hline Cass $\ldots . \ldots \ldots \ldots$ & 2 & ----------- & 19,200 & --------- & 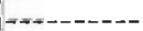 & 19,200 \\
\hline Cedar... & 3 & --- - - - & & & & 38,199 \\
\hline Cerro Gordo ....... & 11 & -ח-ר-n- & 723,988 & 30,775 & & $1,447,868$ \\
\hline Clarke $\ldots \ldots \ldots \ldots$ & 5 & ---------- & -.---_-- & 1,345 & ----- & 2,745 \\
\hline Clay $\ldots \ldots \ldots \ldots$ & 1 & & & & & \\
\hline Clayton $\ldots \ldots \ldots$ & 11 & $-\ldots-\ldots$ & 5,272 & 9,804 & 905 & 15,981 \\
\hline Clinton ... & 10 & ----------- & 15,450 & 1,870 & - & 44,699 \\
\hline Craw ford $\ldots \ldots \ldots$ & 2 & & 6,520 & & ---------- & 6,520 \\
\hline Dallas $\ldots \ldots \ldots \ldots$ & $7^{\prime}$ & 299,407 & 178,488 & & $------\infty n$ & 477,910 \\
\hline
\end{tabular}


TABLE NO. I-CONTINUED

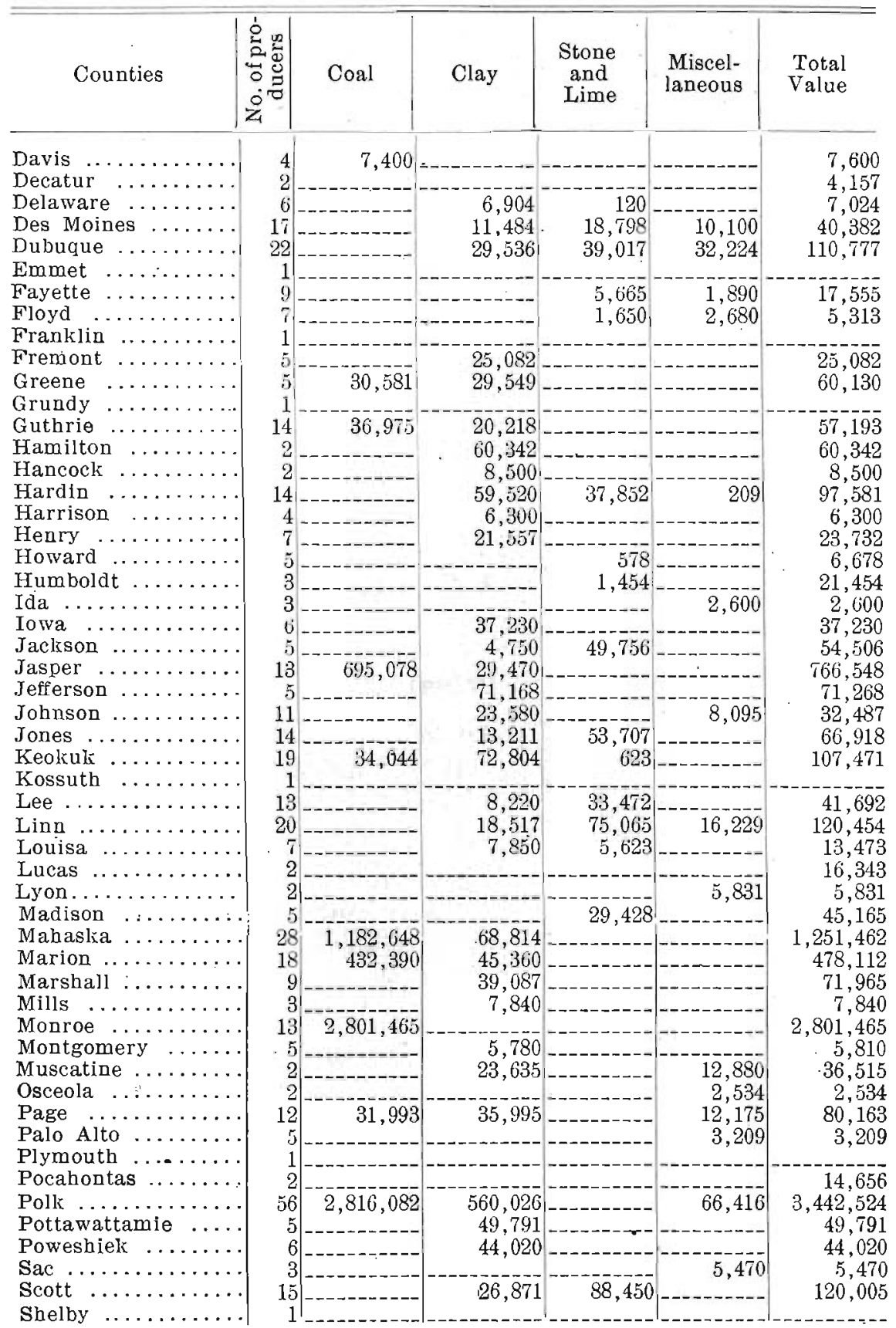


MINERAL PRODUCTION IN IOWA FOR 1908

T.ABLE NO. I-CONTINUED

\begin{tabular}{|c|c|c|c|c|c|c|}
\hline Counties & 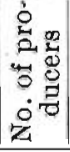 & Coal & Clay & $\begin{array}{l}\text { Stone } \\
\text { and } \\
\text { Lime }\end{array}$ & $\begin{array}{l}\text { Miscel- } \\
\text { laneous }\end{array}$ & $\begin{array}{l}\text { Total } \\
\text { Value }\end{array}$ \\
\hline \multicolumn{7}{|c|}{$\operatorname{Sioux} \ldots \ldots \ldots \ldots$} \\
\hline Story $\quad$.... & 4 & & 37,860 & & & 37,860 \\
\hline Tama ... & & & 75,391 & & & 75,391 \\
\hline Taylor .. & 3 & 33,881 & 9,207 & & & 43,088 \\
\hline Union ..... & 2 & & 33,925 & & & 33,925 \\
\hline Van Buren & 13 & $? 6.964$ & 6,939 & 492 & & 34,395 \\
\hline Wapello $\ldots$ & 21 & 291,079 & 76,434 & 18,150 & 9,365 & 398,028 \\
\hline Washington & ${ }^{8}$ & 16,633 & $19,847$. & & & 36,480 \\
\hline $\begin{array}{l}\text { Washington } \\
\text { Wayne ..... }\end{array}$ & $\begin{array}{r}10 \\
9\end{array}$ & 234.843 & 42,511 & 1,701 & - & 44,212 \\
\hline $\begin{array}{l}\text { Wayne } \\
\text { Webster }\end{array}$ & 30 & $\begin{array}{l}234,840 \\
127,604\end{array}$ & 578,310 & & 567238 & $\begin{array}{r}236,593 \\
\end{array}$ \\
\hline Winnebago & $\begin{array}{c}00 \\
1\end{array}$ & & 010,010 . & & 006,2000 & $1,273,152$ \\
\hline Winnest & 1 & & & & & \\
\hline $\begin{array}{l}\text { Woodbury } \\
\text { Worth }\end{array}$ & 10 & & $224,623 \mid$ & $\ldots$ & 46,370 & 270,993 \\
\hline $\begin{array}{c}\text { worth } \\
\text { wright }\end{array}$ & 8 & & $98,800]$ & & 14.870 & 113 \\
\hline Single Producer & & 17,477 & 232,565 & 50,242 & 806,612 & 143,987 \\
\hline Totals & & $\$ 11,772,228$ & $4,078,627 \mid \$$ & 569,775 & 9,817 & $\$ 18,0 s$ \\
\hline
\end{tabular}

\section{Coal}

The coal production for 1908 shows a shrinkage of nearly six per cent while the average price was about three cents per ton higher, the highest price on record since 1880. Monroe county shows the largest shrinkage and all but two of the leading coal. producing counties show a falling off in production. Polk and Mahaska are the exceptions. The average number of men employed is the largest in the history of the industry in Iowa while the average number of days worked is the lowest since 1897. The output, disposition of product, value, average price per ton, average number of days worked and average number of men employed are given by counties below: 
TABLE NO. II.

COAL PRODUCTION OF IOWA IN 1908, BY COUNTIES.

\begin{tabular}{|c|c|c|c|c|c|c|c|c|c|}
\hline Counties & $\begin{array}{l}\text { Loaded at } \\
\text { mines for } \\
\text { shipment- } \\
\text { short Tons }\end{array}$ & {$\left[\begin{array}{c}\text { Sold tio local } \\
\text { trade and } \\
\text { used by } \\
\text { employes- } \\
\text { Short Tons }\end{array}\right.$} & $\begin{array}{c}\text { Used at } \\
\text { mines for } \\
\text { steam and } \\
\text { heat- } \\
\text { Short Tons }\end{array}$ & $\begin{array}{c}\text { Total } \\
\text { quantity- } \\
\text { Short Tons }\end{array}$ & & $\begin{array}{l}\text { Total } \\
\text { value }\end{array}$ & $\begin{array}{c}\text { Average } \\
\text { price } \\
\text { per ton }\end{array}$ & $\begin{array}{l}\text { A verage } \\
\text { number } \\
\text { of days } \\
\text { worked }\end{array}$ & $\begin{array}{l}\text { A verage } \\
\text { number } \\
\text { of em- } \\
\text { ployes }\end{array}$ \\
\hline Adams $\quad \ldots \ldots \ldots \ldots \ldots \ldots \ldots \ldots$ & 300 & 17,152 & 40 & 17,492 & $\$$ & 42,235 & 2.47 & 141 & 83 \\
\hline Appanoose $\ldots \ldots \ldots \ldots \ldots \ldots$ & $1,101,788$ & 45,901 & 11,492 & $1,159,181$ & & $2,151,905$ & 1.86 & 175 & 4,170 \\
\hline Boone $\ldots \ldots \ldots \ldots \ldots \ldots \ldots \ldots$ & 196,537 & 32,421 & 8,540 & 237,498 & & 461,544 & 1.95 & 184 & 735 \\
\hline Dallas $\ldots \ldots \ldots \ldots \ldots \ldots \ldots \ldots \ldots$ & 160,315 & $\tau, 130$ & 7,140 & 174,585 & & 299,407 & 1.71 & 234 & 407 \\
\hline Davis $\ldots \ldots \ldots \ldots \ldots \ldots \ldots \ldots$ & & 3,700 & & 3,700 & & 7,400 & 2.00 & 146 & 24 \\
\hline Greene $\ldots$ & & 12,531 & 400 & 12,931 & & 30,581 & 2.37 & 223 & 37 \\
\hline Guthrie .. & & 13,179 & & 13,184 & & 36,975 & 2.80 & 170 & 72 \\
\hline Jasper $\ldots \ldots \ldots \ldots \ldots$ & 364,500 & 10,682 & 18,334 & 393,516 & & 695,078 & 1.77 & 233 & 804 \\
\hline Keokuk ... & & 17,206 & 1,095 & 18,301 & & 34,044 & 1.86 & 219 & 31 \\
\hline $\begin{array}{l}\text { Lucas ..... } \\
\text { Mahaska . }\end{array}$ & 755,653 & 34,032 & 19,548 & 809,233 & & $1,182,648$ & 1.46 & 203 & 1,852 \\
\hline Marion .... & 265,401 & 26,896 & 2,290 & 294,587 & & 432,390 & 1.47 & 201 & 646 \\
\hline Monroe ... & $1,864,440$ & 49,074 & 52,105 & $1,965,619$ & & $2,801,465$ & 1.44 & 244 & 3,171 \\
\hline Page...$\ldots \ldots$ & & 11.364 & & 11,364 & & 31,993 & 2.8 & 124 & 59 \\
\hline Polk .......... & $1,3 i 1,356$ & 226,995 & 40,544 & $1,618,895$ & & $2,816,082$ & 1.74 & 228 & 3,129 \\
\hline $\begin{array}{l}\text { Scott } . . . . \\
\text { Taylor } \ldots\end{array}$ & 7,806 & 7,887 & 140 & 15,833 & & 33,881 & 2.12 & 211 & 67 \\
\hline Van Buren & 7,476 & 5,341 & 45 & 12,862 & & 26,964 & 2.10 & 190 & 34 \\
\hline Wapello ... & 126,671 & 55,249 & 2,732 & 184,652 & & 291,079 & 1.58 & 190 & 440 \\
\hline Warren & & 0,670 & & 6,720 & & 16,033 & 2.44 & 145 & 36 \\
\hline Wayne. & 114,074 & 12,053 & 32 & 126,159 & & 234,843 & 1.86 & 234 & 421 \\
\hline Webster $\ldots \ldots \ldots$ & 46,937 & 15,180 & 1,101 & 63,218 & & 127,604 & 2.02 & 183 & 187 \\
\hline Single Producers & 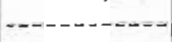 & 9,987 & & 9,987 & & 17,477 & 1.75 & 124 & 34 \\
\hline Total... & $6,363,254$ & 620,630 & 165,633 & $7,149,517$ & $\$$ & $11,772,228$ & 1.65 & 205 & 16,439 \\
\hline $\begin{array}{l}\text { The returns of the United States } \\
\text { Geological Survey are as follows. }\end{array}$ & $6.345,590$ & 650,481 & 165,239 & $7,161,310$ & & $11,706.402$ & 1.63 & 214 & 16,021 \\
\hline
\end{tabular}


The table given herewith presents a fair picture of the Iowa coal industry during the past ten years:

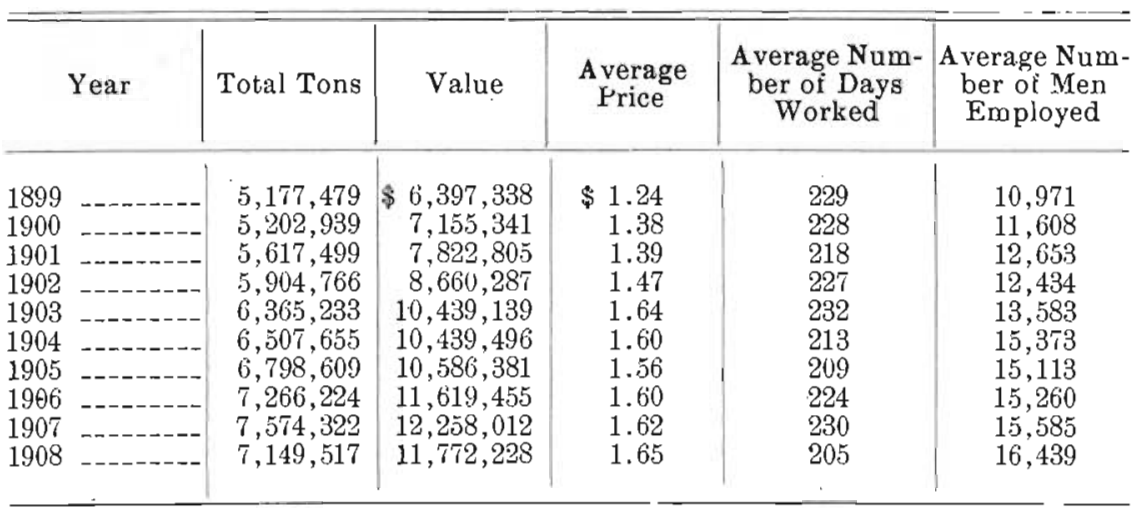

The ten leading producer's during 1907 according to the authority of the United States Geological Survey were as follows:

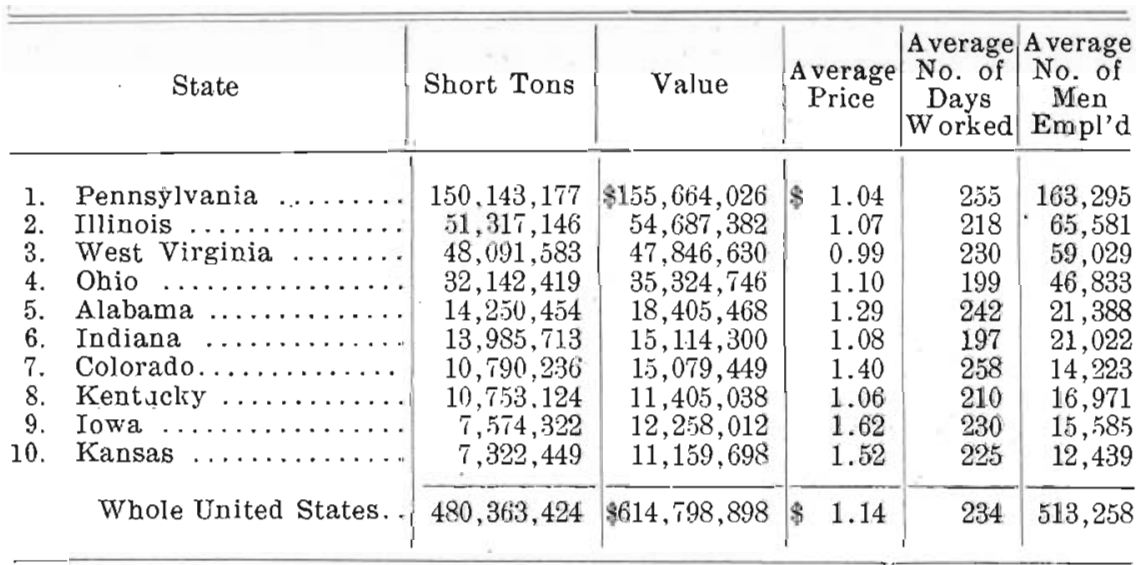

The following table based upon figures given by the United. States Geological Survey gives the production for the ten states which were the leaders in output of coal in 1908. It will be seen that Iowa maintains her rank of the preceding year although there are some changes among other states. As compared with the preceding year there is a sharp decline, both in quantity produced and in spot value: 
TEN IEADING PRODUCING STATES IN 1908.

\begin{tabular}{|c|c|c|}
\hline & State & Short Tons \\
\hline 1. & Pennsylvania & $117,179,527$ \\
\hline 2. & Illinois $\ldots .$. & . $47,659,690$ \\
\hline 3. & West Virginia .... & $41,897,843$ \\
\hline 4. & Ohio $\ldots \ldots \ldots \ldots \ldots$ & . $26,270,639$ \\
\hline 5. & Indiana $\ldots \ldots \ldots \ldots$ & . $12,314,890$ \\
\hline 6. & Alabama $\ldots \ldots \ldots$ & $11,604,593$ \\
\hline 7. & Kentucky $\ldots \ldots \ldots \ldots$ & . $10,246,553$ \\
\hline 8. & Colorado $\ldots \ldots \ldots \ldots$ & - $\quad 9,634,973$ \\
\hline 9. & Iowa $\ldots \ldots \ldots \ldots \ldots$ & $7,161,310$ \\
\hline 10. & Kansas $\ldots \ldots \ldots$ & $6,245,508$ \\
\hline
\end{tabular}

State Value

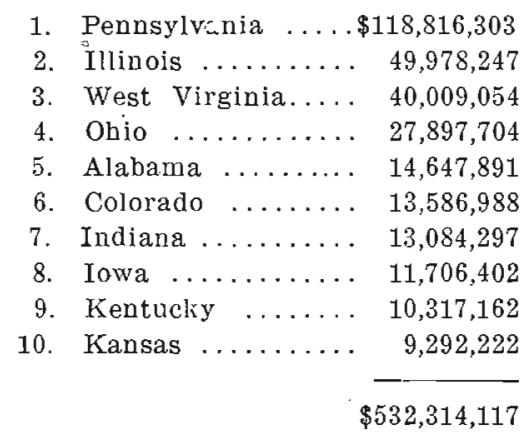

The outlook for 1909 is for an increase in production and a slight falling off in average price per ton.

\section{Clay Products}

The value of clay products marketed in 1908 shows a splendid growth of approximately ten per cent. This growth is recorded in spite of the fact that there was a falling off in production of the majority of the common clay products. Iowa holds her position in first place as a producer and user of drain tile and doubled her output of sewer pipe during the year. Mason City in Cerro Gordo county is the greatest center for the manufacture of drain tile in the United States, and for that matter in the world. Eight plants, fully equipped and up-to-date, are in operation at the present time. Webster county is rapidly coming to the front in the manufacture of hollow ware, including drain tile, sewer pipe and hollow building block. 
The clay products for 1908 were distributed as follows:

\begin{tabular}{|c|c|c|c|c|c|}
\hline \multirow{2}{*}{ Article } & \multirow[t]{2}{*}{ - } & \multirow{2}{*}{$\begin{array}{l}\text { Quantity in } \\
\text { Thousands }\end{array}$} & \multirow{2}{*}{ Value } & \multicolumn{2}{|c|}{$\underset{1907}{\text { Compared With }}$} \\
\hline & & & & $\begin{array}{l}\text { Incr'ase } \\
\text { per cent }\end{array}$ & $\begin{array}{l}\text { Decrase } \\
\text { per cent }\end{array}$ \\
\hline \multirow{2}{*}{\multicolumn{2}{|c|}{$\begin{array}{l}\text { Common Brick } \ldots \ldots \ldots \ldots \ldots \ldots \\
\text { Paving Brick and Block. }\end{array}$}} & 135,678 & 896,890 & & $17+$ \\
\hline & & 16,672 & 185,112 & $\ldots$ & 17 \\
\hline \multicolumn{2}{|c|}{ Face Brick ................. } & 7,900 & 86,232 & & $10+$ \\
\hline \multicolumn{2}{|c|}{ 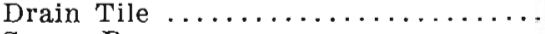 } & -_-_-_-_-_- & $2,522,363$ & $25+$ & $\ldots$ \\
\hline \multicolumn{2}{|c|}{ Sewer Pipe $\ldots \ldots \ldots \ldots \ldots \ldots \ldots \ldots$} & & 211,044 & $104+$ & \\
\hline \multicolumn{2}{|c|}{$\begin{array}{l}\text { Building Block } \ldots \ldots \ldots \ldots \ldots \ldots \ldots \ldots \ldots \\
\text { Pottery } \ldots \ldots \ldots \ldots \ldots \ldots \ldots\end{array}$} & $-\ldots--$ & 129,003 & & $27+$ \\
\hline Pottery $\ldots \ldots \ldots$ & & & 18,710 & & $1-$ \\
\hline \multicolumn{2}{|l|}{ Miscellaneous* } & & 29,273 & $140+$ & \\
\hline \multicolumn{2}{|l|}{ Totalt.... } & 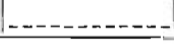 & \$ $4.078,627$ & $10-1$ & 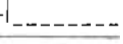 \\
\hline
\end{tabular}

*Includes raw clay mined and sold, $\$ 3,690$.

$\dagger$ The returns of the United States Geological Survey give the total as $\$ 4,073,187$. The figures for pottery and raw clay are as above.

TABLE NO. TIT.

VALUE OF IOWA CLAY PRODUCTS FOR 1908, TABULATED BY COUNTIES.

\begin{tabular}{l|r|r|r|r|r|r}
\hline \hline & & & \\
& \\
& \\
&
\end{tabular}


TABLE NO. III-CONTINUED

\begin{tabular}{|c|c|c|c|c|c|c|c|}
\hline Counties & 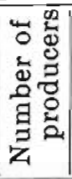 & $\begin{array}{c}\text { Com'on } \\
\text { Brick }\end{array}$ & $\begin{array}{l}\text { Paving } \\
\text { Brick or } \\
\text { Block }\end{array}$ & $\begin{array}{l}\text { Face } \\
\text { Brick }\end{array}$ & $\begin{array}{c}\text { Drain } \\
\text { Tile }\end{array}$ & $\begin{array}{l}\text { Miscel- } \\
\text { laneous }\end{array}$ & $\begin{array}{l}\text { Total } \\
\text { Value }\end{array}$ \\
\hline Delaware ........ & 3 & 1,600 & & & 2,304 & 3,000 & 6,904 \\
\hline Des Moines ........ & 3 & 5,901 & & 83 & 5,500 & & 11,484 \\
\hline Dubuque $\ldots \ldots \ldots$ & 3 & 29,536 & & & 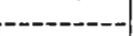 & & 29,536 \\
\hline Emmet ......... & 1 & -------- & & & & & -------- \\
\hline Fayette ........ & 1 & & & & ------ & & ------- \\
\hline 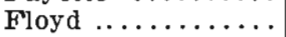 & 1 & & & & & & \\
\hline Fremont ......... & 5 & 4,999 & & & & 20,083 & 25,082 \\
\hline Greene $\ldots . . .$. & 2 & 500 & & & 29,049 & & 29,549 \\
\hline Grundy $\quad \ldots \ldots \ldots$ & 1 & & & & & & \\
\hline Guthrie ......... & 3 & 2,554 & & & 13,418 & 4,246 & 20,218 \\
\hline Hamilton...$\cdots$ & 2 & 11,142 & & & 49,200 & $-\infty-1$ & 60,342 \\
\hline Hancock $\ldots . . .$. & 2 & & & & 8,500 & & 8,500 \\
\hline Hardin .......... & 5 & 500 & & & 58,520 & 500 & 59,520 \\
\hline Harrison ........ & 4 & 6,300 & & & & & 6,300 \\
\hline Heniry $\ldots \ldots \ldots$. & 6 & 1,707 & & & 19,850 & & 21,557 \\
\hline Howard ......... & 1 & & & & & & $-\cdots$ \\
\hline Humboldt ....... & 1 & & & & & & \\
\hline Ida $\ldots \ldots \ldots \ldots$ & 1 & & & & & & \\
\hline Iowa $\ldots \ldots \ldots$ & 6 & $-10,750$ & 900 & & 25,580 & & 37,230 \\
\hline Jackson $\ldots \ldots \ldots$ & 2 & 550 & & & 4,200 & & 4,750 \\
\hline Jasper $\ldots \ldots \ldots$ & 6 & 13,200 & & & 16,020 & 250 & 29,470 \\
\hline Jefferson $\ldots . .$. . & 4 & 12,005 & & & 59,163 & $\ldots$ & 71,168 \\
\hline Johnson $\ldots \ldots \ldots$ & 4 & 15,080 & & & 8,500 & & 23,580 \\
\hline Jones ........... & 3 & 2,393 & & & 10,818 & & 13,211 \\
\hline Keokuk ........ & 10 & 5,712 & & & 67,092 & & 72,804 \\
\hline Kossuth ........ & 1 & & & & & & \\
\hline Lee $\ldots \ldots \ldots \ldots$ & 3 & 6,850 & & 250 & 1,120 & ב-ב_. & 8,220 \\
\hline $\operatorname{Lin} n \ldots \ldots \ldots \ldots$ & 6 & 8,553 & & ------- & 9,964 & - & 18,517 \\
\hline Louisa $\ldots . . . \ldots$. & 2 & 1,250 & & & 6,600 & & 7,850 \\
\hline Lucas ...... & 1 & & & & & & \\
\hline Madison...$\ldots \ldots$ & 1 & & & & & & \\
\hline Mahaska ........ & 4 & 14,213 & 25,000 & 1,600 & 28,001 & & 68,814 \\
\hline Marion .......... & 3 & 18,260 & - & --- & 22,800 & 4,300 & 45,360 \\
\hline Marshall ........ & 8 & 7,550 & & 5,967 & 20,570 & 5,000 & 39,087 \\
\hline Mills $\ldots \ldots \ldots \ldots$ & 3 & 7,840 & & & & & 7,840 \\
\hline Montgomery ...... & 4 & 3,830 & & & 1,700 & . 250 & 5,780 \\
\hline Muscatine $\ldots \ldots$. & 9 & 14,024 & & & 1,500 & 8,111 & 23,635 \\
\hline Page........ & 5 & 27,775 & & & 8,220 & & 35,995 \\
\hline Palo Alto $\ldots . . .$. & 1 & ---- & $\ldots-\infty$ & & $-\ldots$ & & ------- \\
\hline Plymouth $\ldots . .$. & 1 & 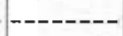 & $\cdots-$ & & $-\infty-\infty$ & & 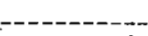 \\
\hline Pocahontas ...... & 1 & & & & & & \\
\hline Polk .......... & 15 & 115,579 & 128,415 & 22,135 & 164,449 & 129,448 & 560,026 \\
\hline Pottawattamie .... & 5 & 49,541 & & - & 250 & & 49,791 \\
\hline Poweshiek $\ldots \ldots$. & 6 & 6,364 & & & 37,656 & & 44,020 \\
\hline Scott $\ldots \ldots \ldots \ldots$ & 3 & 12,890 & 650 & 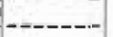 & 4,221 & 9,110 & 26,871 \\
\hline Shelby $\ldots \ldots \ldots \ldots$ & 1 & & & & & & \\
\hline Sioux ... & & & & & & & \\
\hline Story $\ldots \ldots \ldots \ldots$ & 4 & 6,100 & 200 & 360 & 31,200 & & 37,860 \\
\hline Tama .......... & 6 & 27,205 & & 16,670 & 31.516 & & 75,391 \\
\hline Taylor ......... & 2 & 4.226 & 111 & $\mid-\ldots$ & 4.860 & & 9.207 \\
\hline Union $\ldots \ldots \ldots \ldots$ & 2 & 7,875 & $-\ldots-1$ & & 20,500 & 5,550 & 33.925 \\
\hline Van Buren $\ldots \ldots$ & 3 & 2,861 & - & & 4,078 & & 6.939 \\
\hline
\end{tabular}


TABLE NO. III-CONTINUED

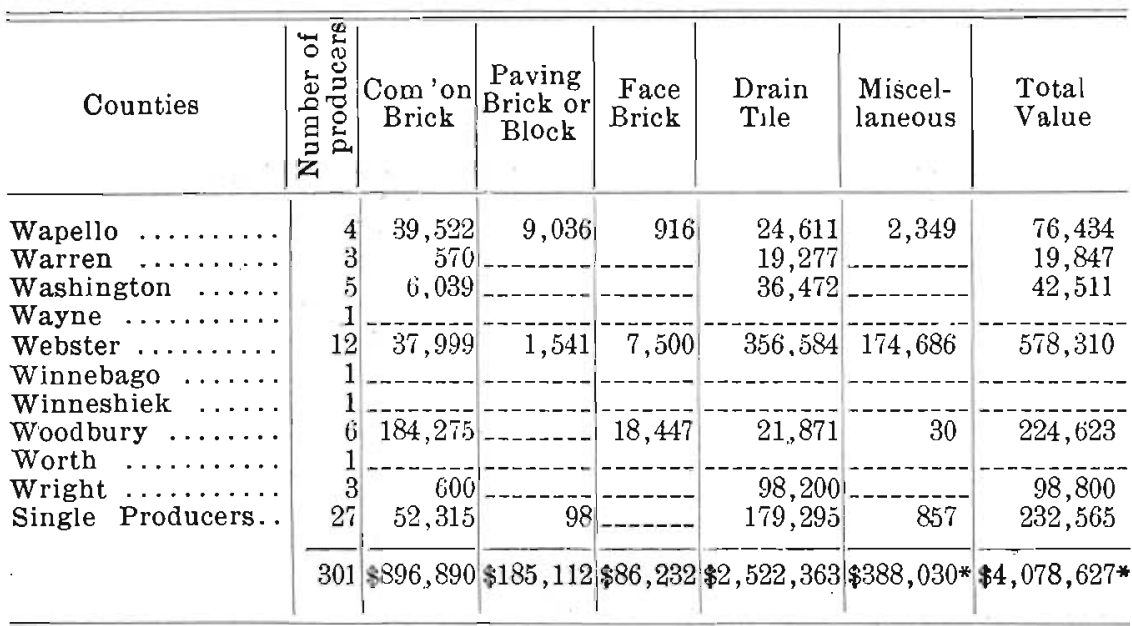

*Includes pottery and raw clay mined and sold.

Iowa ranked ninth in the total production for 1907, maintaining her rank and showing an increase in production over the preceding year of 7.49 per cent against a decrease of 1.30 per cent for the whole United.States. The ten leading producers, with number of firms in operation, value and percentage of total products, are given below, as tabulated by the United States Geological Survey:

\begin{tabular}{|c|c|c|c|}
\hline State & $\begin{array}{l}\text { No. of } \\
\text { Operat- } \\
\text { ing firms } \\
\text { reporting }\end{array}$ & Value & $\begin{array}{c}\text { Percentage } \\
\text { of total } \\
\text { Product }\end{array}$ \\
\hline Ohio $\ldots . . . \ldots \ldots \ldots$. & 736 & $30,340,830$ & 19.09 \\
\hline Pennsylvania ......... & 487 & $20,291,621$ & 12.77 \\
\hline New Jersey $\ldots \ldots \ldots \ldots \ldots \ldots \ldots \ldots \ldots$ & 165 & $16,005,460$ & 10.07 \\
\hline New York .................... & 417 & $13,220,489$ & 8,32 \\
\hline Illinois $\quad \ldots \ldots \ldots \ldots \ldots \ldots \ldots \ldots \ldots \ldots$ & 247 & $11,772,874$ & 7.41 \\
\hline Indiana $\ldots \ldots \ldots \ldots \ldots \ldots \ldots \ldots \ldots$ & 172 & 6.898 .871 & 4.34 \\
\hline Missouri $\ldots \ldots \ldots \ldots \ldots \ldots \ldots \ldots$ & 392 & $6,858,124$ & 4.32 \\
\hline California $\ldots \ldots \ldots \ldots \ldots \ldots \ldots \ldots \ldots$ & 118 & $5,740,537$ & 3.61 \\
\hline Iowa $\ldots \ldots \ldots \ldots \ldots \ldots \ldots \ldots \ldots \ldots$ & 276 & $3,728,785$ & 2.35 \\
\hline 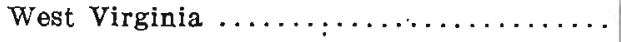 & 63 & $3,640,387$ & 2.29 \\
\hline Total for United States............. & 5,536 & $\$ 158,942,369$ & 100.00 \\
\hline
\end{tabular}




\section{Pottery}

The production of pottery for the year 1908 shows but little change when compared with that for 1907. The output for 1908 was as follows:

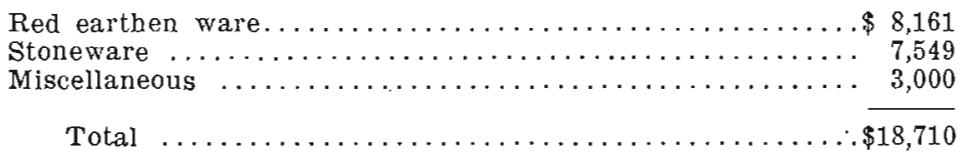

An increased output is expected for the present year owing to the enlargement of the plant at Ottumwa.

\section{Clay}

The production and sale of raw clay during 1908 was less than for the preceding year. This is shown not so much in the diminished tonnage as in the lower prices received, especially for fire clay. These facts are shown in the following table:

\begin{tabular}{|c|c|c|c|c|c|}
\hline & Kind & 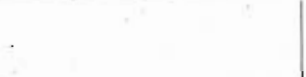 & $\begin{array}{l}\text { Quantity in } \\
\text { Short Tons }\end{array}$ & & Value \\
\hline $\begin{array}{l}\text { Fire Clay ... } \\
\text { Brick Clay ... } \\
\text { Miscellaneous }\end{array}$ & $\ldots$ & 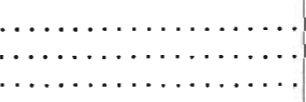 & $\begin{array}{r}6,234 \\
2,400 \\
500\end{array}$ & $\$$ & $\begin{array}{r}1,990 \\
1,200 \\
500\end{array}$ \\
\hline Total ... & & & 9,134 & $\$$ & 3,690 \\
\hline
\end{tabular}

\section{Stone}

The quarry production for 1908 shows a falling off of about twelve and one-half per cent. The greatest shrinkage is in stone used for building purposes. The amount of crushed stone used for concrete shows a marked increase. The production for the year was distributed as follows:

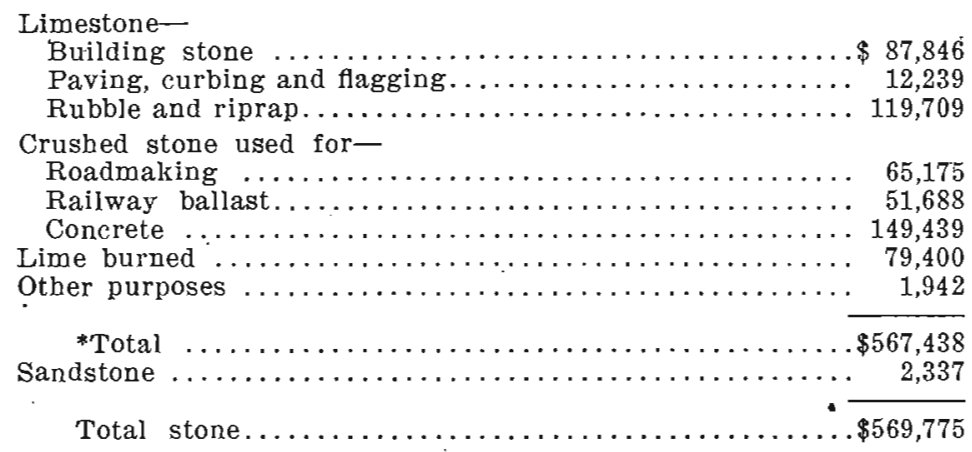

*The returns of the r Inited States Geological Survey give the total output of limestone as $\$ 610,345$. The lime produced is valred t $\$ 79,400$, a $\$$ above. 
TABLE NO. IV.

PRODUCTION OF LIMESTONE IN 1908.

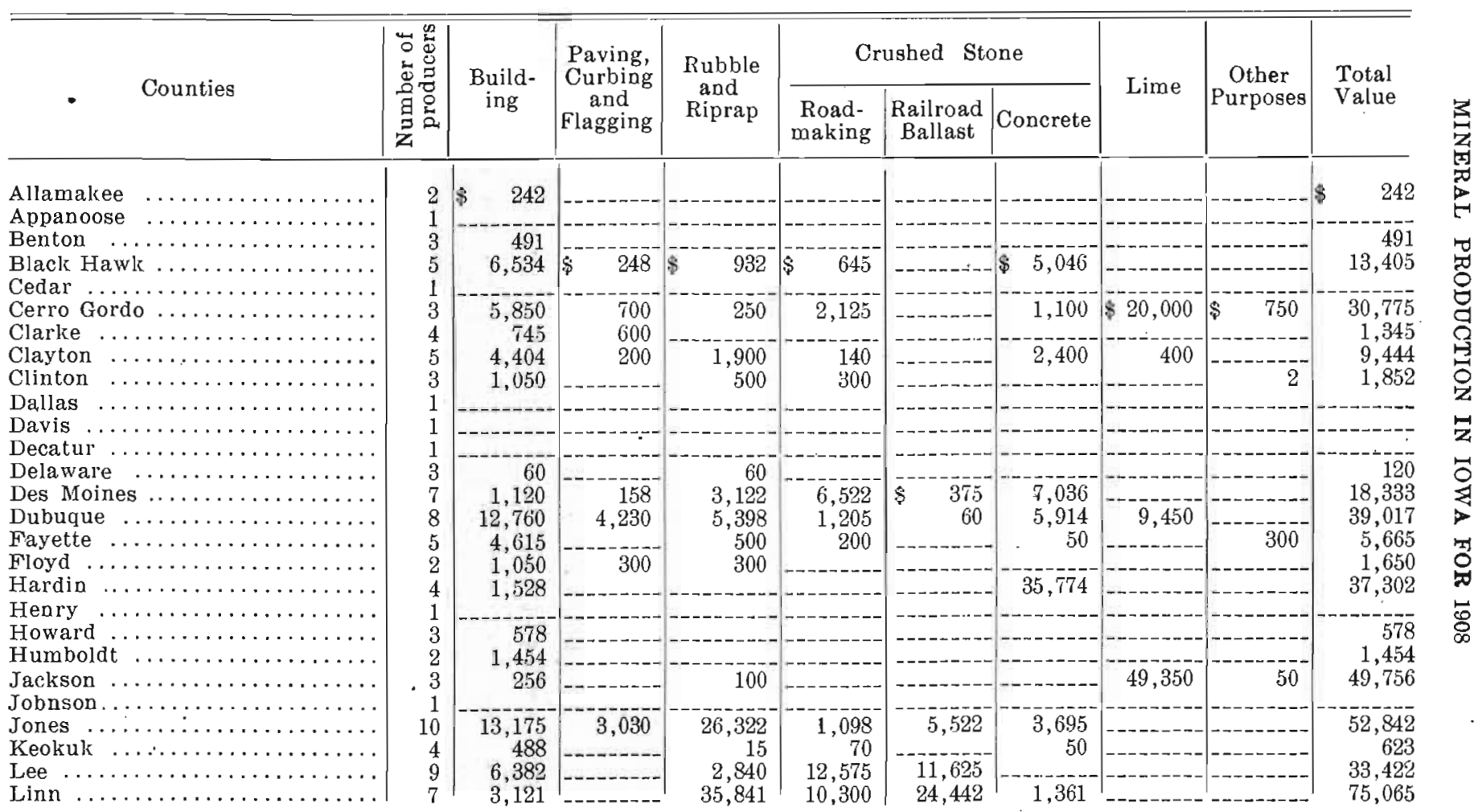




\begin{tabular}{|c|c|c|c|c|c|c|c|c|c|c|}
\hline $\begin{array}{l}\text { Louisa } \ldots \ldots \ldots \ldots \ldots \ldots \ldots \ldots \\
\text { Madison }\end{array}$ & 5 & 2,600 & & 3,023 & 5.900 & 7700 & 13,628 & & & $\begin{array}{r}5,623 \\
29,428\end{array}$ \\
\hline $\begin{array}{l}\text { Madison } \ldots \ldots \ldots \ldots \ldots \ldots \ldots \ldots \\
\text { Marshall } \ldots \ldots \ldots \ldots \ldots \\
\ldots\end{array}$ & $\begin{array}{l}4 \\
1\end{array}$ & & & & 5,900 & 7,100 & 13,628 & & 500 & \\
\hline Pocahontas...$\ldots \ldots \ldots \ldots \ldots \ldots$ & & & & & & & & & & \\
\hline Scott $\ldots \ldots \ldots \ldots \ldots \ldots \ldots \ldots$ & $10^{2}$ & 6,419 & 2,696 & 5,399 & 23,373 & 1,950 & 48,584 & & -------- & 88,421 \\
\hline Van Buren $\ldots \ldots \ldots \ldots \ldots \ldots \ldots$ & 6 & 192 & $\ldots$ & 300 & & & & & & \\
\hline Wapello $\ldots \ldots \ldots \ldots \ldots \ldots \ldots$ & 3 & 6,800 & -.- & 5,120 & $---1-1-$ & --- & 0,230 & & & 18,150 \\
\hline Washington...$\ldots \ldots \ldots \ldots \ldots$ & 5 & 496 & $-----1=$ & 125 & -.-- & ..--- & 1,050 & & 30 & 1,701 \\
\hline Single Producers............. & 9 & 3,736 & 77 & 27,662 & 722 & 14 & 17,521 & 200 & 310 & 50,242 \\
\hline . & 131 & $\$ 87,846$ & $\$ 12,239$ & $\$ 119,709$ & $\$ 65,175$ & $\$ 51,688$ & $\$ 149,439$ & 79,400 & $\$ 1,942$ & 567,438 \\
\hline
\end{tabular}


The outlook for the stone industry is not encouraging save in the line of crushed stone for road work and concrete. The growth in the use of cement and cement products is reducing the demand for stone and brick for structural purposes.

\section{Sand and Gravel}

According to the reports received the production of sand and gravel for 1908 more than doubled the output for 1907 . The records for both years are more or less incomplete. During the latter year the majority of commercial producers responded. The production reported by ninety-four producers representing. twenty-nine counties may be classified as follows:

\begin{tabular}{|c|c|c|c|}
\hline Kind & $\begin{array}{l}\text { Quantity in } \\
\text { cubic yards }\end{array}$ & & Value \\
\hline 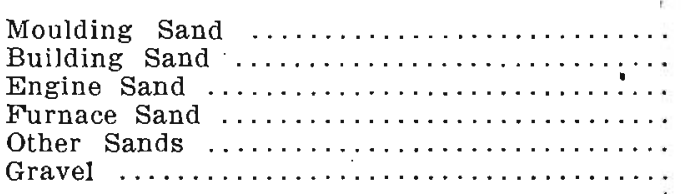 & $\begin{array}{r}3,635 \\
388,644 \\
20,826 \\
2,000 \\
36,146 \\
167,559\end{array}$ & $\$$ & $\begin{array}{r}5,054 \\
205,121 \\
5,811 \\
875 \\
14,039 \\
55,544\end{array}$ \\
\hline 'Total $\ldots \ldots \ldots \ldots \ldots \ldots \ldots \ldots \ldots \ldots$ & 618,810 & $\$$ & 286,444 \\
\hline
\end{tabular}

The value of the output reported for 1907 was $\$ 110,501$.

Table $V$ shows the value of sand and gravel produced in Iowa in 1908 by counties: 
TABLE $V$.

SAND AND GRAVEL PRODUCTION IN IOWA IN 1908 BY COUNTIES.

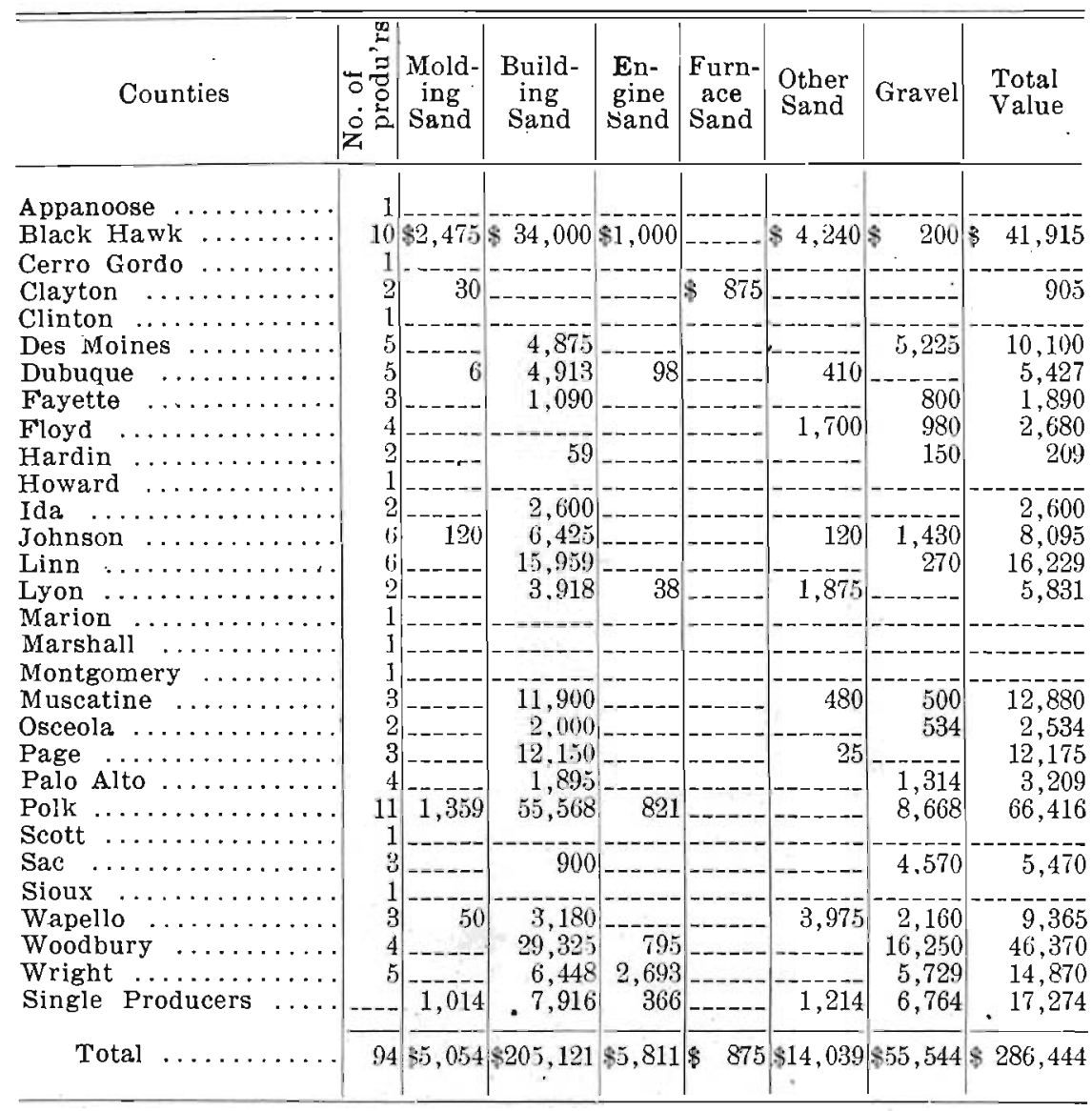

\section{Gypsum}

The production of gypsum and gypsum products for 1908 was less than for the preceding year. The statistics of the industry for the year 1908 were as follows: 


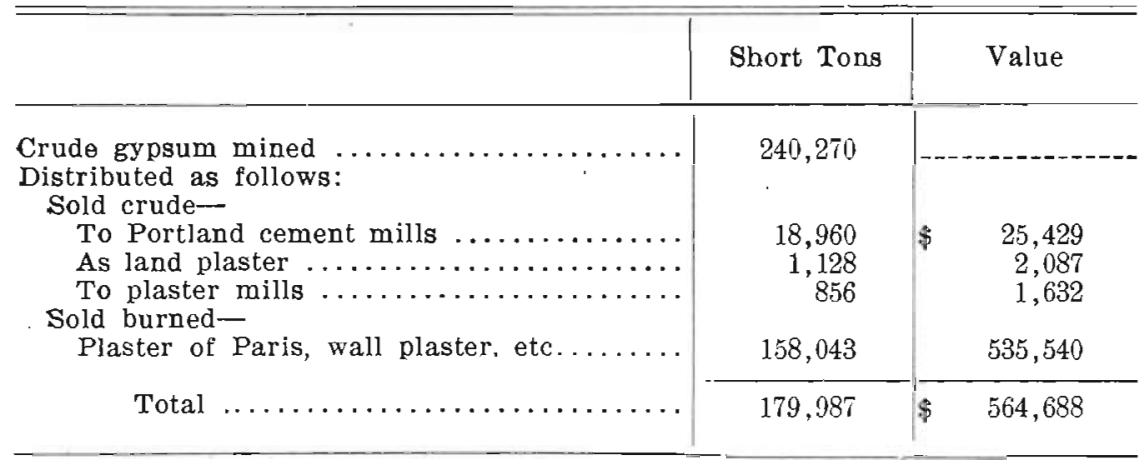

The production of crude gypsum for 1907 was 251,874 short tons and was distributed as crude gypsum 18,834 tons valued at $\$ 29,115$ and burned gypsum 162,965 tons valued at $\$ 701,268$. The price per ton was considerably lower for 1908 than for the preceding year.

\section{Mineral Water}

The amount of mineral water produced and sold was the largest in the history of the industry in Iowa. Five counties reported sales during the year. The springs at Colfax in Jasper county are by far the largest producers. The amount and value of the water sold were as follows:

\begin{tabular}{|c|c|c|c|}
\hline & $\begin{array}{l}\text { Quantity in } \\
\text { gallons }\end{array}$ & & Value \\
\hline $\begin{array}{l}\text { Medicinal } \ldots \ldots \ldots \ldots \ldots \ldots \ldots \ldots \ldots \ldots \ldots \ldots \ldots \ldots \ldots \ldots \ldots \\
\text { Table } \ldots \ldots \ldots \ldots \ldots \ldots \ldots \ldots\end{array}$ & $\begin{array}{l}381,500 \\
102,000\end{array}$ & $\$$ & $\begin{array}{l}41,650 \\
13,700\end{array}$ \\
\hline Total sold & 483,500 & $\$$ & 55,350 \\
\hline
\end{tabular}

Used for soft drinks 317,500 gallons, not included in above figures.

The sales for 1907 amounted to 127,200 gallons valued at $\$ 30,500$.

\section{Lead and Zinc}

On account of the slump in the price of pig lead the amount of lead ore produced and sold in the Dubuque region was much less than for the preceding year. The sale of zinc ores accumu- 
lated during several years brought up the zinc figures. The amount of ore sold during the year was as follows:

\begin{tabular}{|c|c|c|c|}
\hline$\cdot$ & $\begin{array}{l}\text { Quantity in } \\
\text { pounds }\end{array}$ & & Value \\
\hline 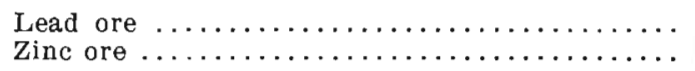 & $\begin{array}{l}124,900 \\
950,000\end{array}$ & $\$$ & $\begin{array}{r}3,614 \\
23,183\end{array}$ \\
\hline
\end{tabular}

The outlook for the present year is not promising unless the prices of pig lead and spelter increase. Some lead is being mined and held in stock. Prospecting and development work cońtinue in a small way.

\section{Sand-lime Brick}

The sand-lime brick industry shows a falling off both in quantity and value of output. There was no change in the number of plants. The product was distributed as follows:

\begin{tabular}{|c|c|c|c|}
\hline & $\begin{array}{l}\text { Number in } \\
\text { thousands }\end{array}$ & & Value \\
\hline 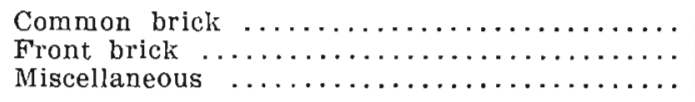 & $\begin{array}{r}4,701 \\
535 \\
-\end{array}$ & $\$$ & $\begin{array}{r}33,784 \\
5,223 \\
3,874\end{array}$ \\
\hline 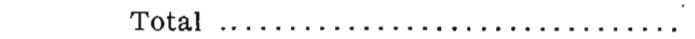 & 5,236 & s & 42,881 \\
\hline
\end{tabular}

\section{Portland Cement}

Iowa for the first time appears as a producer of Portland cement. The Northwestern States Portland Cement Company of Mason City was the only plant in operation during the year. The plant of the Iowa Portland Cement Company of Des Moines is approaching completion and will probably be a producer during the present year. The Hawkeye Portland Cement Company has made but little progress during the year. The price of cement to Iowa consumers is the lowest it has ever been. The Lehigh Portland Cement Company has purchased property in the vicinity of Mason City and contemplates putting up a plant in the near future. 


\section{Iron}

The Missouri Iron Company continued development work during 1908 and perfected its plant for handling and treating ore. Ore on a commercial basis was not produced during the year.

\section{Peat}

But little progress was made during the year toward the production and utilization of peat on a commercial scale. The plant installed near Fertile in Worth county was operated during a portion of the year but rather in an experimental way. 